\title{
NOTES ON TRANSLATION AND TRANSLITERATION
}

Throughout the book, I have followed the standard spelling for Bangla words as they are written in Bangladesh. I have avoided the International Phonetic Alphabet for the ease in reading for both Bengali and non-Bengali readers.

All translations from Bangla, unless otherwise noted, are mine. 
This page intentionally left blank 